\title{
Experiencias
}

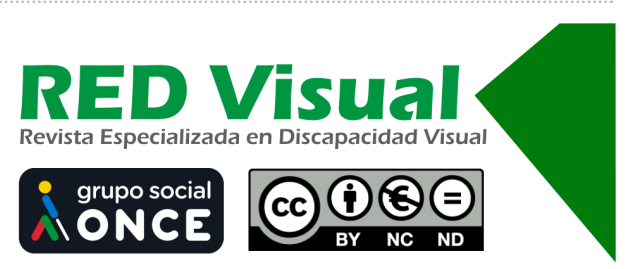

Recepción: 31-12-2020

Aceptación: 06-05-2021

\section{Nuevas tecnologías, voluntariado y participación activa: tres claves para activar a las personas ${ }^{1}$}

\author{
New technologies, volunteering and active participation: \\ three keys to activating people
}

\author{
J. M. Fernández Granda, M. Malgor Vázquez, \\ M. D. Rodríguez Miranda
}

\section{Resumen}

Las nuevas tecnologías son fuente de oportunidades para cualquier persona, más aún con discapacidad visual. No tener acceso a ellas genera importantes brechas sociales, culturales y económicas. Por ello, resulta necesario desarrollar metodologías que faciliten formación adecuada en competencias digitales. En la Delegación Territorial de la Organización Nacional de Ciegos Españoles (ONCE) de Asturias hemos desarrollado un programa para facilitar el uso de tecnología mediante la interacción social y la colaboración, orientar en su uso para mejorar la calidad de vida y la autonomía personal y dar una formación continua. Hemos utilizado metodologías participativas y trabajo multidisciplinar, coordinando voluntariado, organizando actividades de ocio y utilizando nuevas tecnologías. Así, pudieron atenderse satisfactoriamente necesidades formativas de más personas, iniciando esta andadura con nueve usuarios en abril de 2017 hasta alcanzar 66 en octubre de 2020. Durante este tiempo, hemos logrado promover el trabajo en equipo, aumentar la implicación y participación de las personas en actividades socioculturales y facilitar formación continua e intercambio de criterios.

1 Trabajo galardonado con el primer premio en la categoría General del v Concurso de Experiencias de Innovación y Buenas Prácticas en Servicios Sociales de la ONCE, presentado bajo el mismo título.

Fernández, J.M., Malgor, M., y Rodríguez, M.D. (2021). Nuevas tecnologías, voluntariado y participación activa: tres claves para activar a las personas. RED Visual: Revista Especializada en Discapacidad Visual, 78, 55-68. https://doi.org/10.53094/SNSM7010. 


\title{
Palabras clave
}

Tecnologías. Discapacidad. Voluntariado. Participación, Coordinación interdisciplinar.

\begin{abstract}
New technologies are a source of opportunities for everyone, even more so for the visually impaired. A lack of access to them creates significant social, cultural and economic gaps. It is therefore necessary to develop methodologies that provide adequate training in digital skills. At the Asturias Territorial Branch of the Spanish National Organisation for the Blind (ONCE) we have developed a programme to facilitate the use of technology through social interaction and collaboration, to guide its use to improve quality of life and personal autonomy and to provide continuous training. We have used participatory methodologies and multidisciplinary work, coordinating volunteers, organising leisure activities and using new technologies. The training needs of more people could thus be successfully addressed, starting with nine users in April 2017 and reaching 66 in October 2020. During this time we have succeeded in encouraging teamwork, increasing people's involvement and participation in socio-cultural activities, and providing continuous training and exchanges of opinions.
\end{abstract}

\section{Key words}

Technologies. Disability. Volunteers. Participation, Interdisciplinary coordination.

\section{Introducción}

Todo es muy difícil antes de ser sencillo.

Thomas Fuller

En medio de la dificultad, yace la oportunidad.

Einstein

Internet se ha convertido en el medio de comunicación por excelencia, en una revolución que afecta a nuestra vida cotidiana por ser herramienta común desde la que ciudadanos de todo el mundo interaccionan, convirtiendo su uso en imprescindible.

Hace años muchos, expertos vaticinaban las posibilidades de universalizar el conocimiento y de globalizar las comunicaciones, pero se podía intuir el alcance real que

Fernández, J.M., Malgor, M., y Rodríguez, M.D. (2021). Nuevas tecnologías, voluntariado y participación activa: tres claves para activar a las personas. RED Visual: Revista Especializada en Discapacidad Visual, 78, 55-68. https://doi.org/10.53094/SNSM7010. 
determinaría nuestras vidas: estudiar o trabajar entraba dentro de lo comprensible, pero comprar, vender, pagar, hacer gestiones bancarias, administrativas, comunicativas o deportivas, relacionarse con los demás, aun siendo posible, se consideraba poco menos que ciencia ficción o tecnología de difícil manejo dirigida a un número reducido de ciudadanos formados y expertos en informática.

Esta revolución provoca un cambio de paradigma cultural que convierte en analfabetos digitales a personas con sobradas competencias intelectuales que no sean capaces de utilizar nuevos soportes y herramientas informáticas. La incorporación de las tecnologías de la información y la comunicación (TIC) es un fenómeno social heterogéneo que ha provocado cambios significativos en el acceso al conocimiento. El hecho de no tener acceso a las mismas dificulta la igualdad de oportunidades y genera una importante brecha cultural, económica y social (Pérez y Sarrate, 2011).

Quizá uno de los soportes fundamentales de las TIC es el teléfono móvil. Apareció a finales del siglo pasado y evolucionó hasta convertirse en la primera década del 2000 en smartphone, popularizándose su comercialización y uso a partir del 2010.

Un teléfono inteligente combina dos funciones: teléfono móvil y ordenador de bolsillo. Sus principales características son la pantalla táctil, que reemplaza teclados físicos tradicionales, y la pantalla visual pequeña, lo que, a priori, podría dificultar el acceso a personas con ceguera o déficit visual grave.

Su capacidad multitarea con soportes de correo electrónico y acceso a internet vía wifi o redes LTE, 2G, 3G, 4G o 5G; funciones multimedia, como cámara de fotos, reproducción de audio y de videos; agenda; administración de contactos; control del hogar conectado; GPS, y la posibilidad de crear documentos en varios formatos abren un mundo de posibilidades a todos los usuarios con independencia de sus capacidades, siempre y cuando utilicen los soportes y herramientas de accesibilidad adecuados, pudiendo administrar y gestionar sus acciones cotidianas desde uno o varios terminales.

Hoy sabemos con seguridad que las nuevas tecnologías son fuente de oportunidades, especialmente para mayores y personas con discapacidad, pudiendo contribuir en múltiples áreas para la mejora de su calidad de vida (Piattini, 2012; Fernández et al., 2016). Estas oportunidades que ofrecen las tecnologías pueden aglutinarse en cinco categorías:

Fernández, J.M., Malgor, M., y Rodríguez, M.D. (2021). Nuevas tecnologías, voluntariado y participación activa: tres claves para activar a las personas. RED Visual: Revista Especializada en Discapacidad Visual, 78, 55-68. https://doi.org/10.53094/SNSM7010. 
- Informativas.

- Comunicativas.

- Transaccionales.

- Administrativas.

- De entretenimiento.

Los estudios realizados al respecto concluyen que optimizan la calidad de vida de los mayores y contribuyen a su envejecimiento activo, si bien, su máximo aprovechamiento precisa de programas de «e-Inclusión» y metodologías que aproximen internet, facilitándoles una formación en competencias digitales (Llorente-Barroso et al., 2015; Fernández et al., 2016).

Nosotros hemos llevado a cabo un programa con dichas características orientado hacia el colectivo de personas afiliadas a la ONCE, no solamente mayores, aunque, como se contará en las próximas páginas, conforman un grupo especialmente receptivo, y es precisamente lo que trataremos de exponer en el presente trabajo.

Para llevar a cabo este programa, se ha partido de la base de las teorías del aprendizaje actuales, que reconocen la importancia de las relaciones sociales y la interacción con el otro al adquirir conocimiento. Saber trabajar en grupo para conseguir objetivos comunes aparece como una competencia transversal de aprendizaje en todos los niveles de enseñanza (García-Valcárcel et al., 2012).

Por ello, se ha buscado crear los entornos sociales para la interacción donde las tecnologías se conviertan en un instrumento idóneo. En este sentido, funcionan como medio para la interacción social y, a su vez, la interacción social funciona como medio para la formación en tecnologías.

La importancia en competencias digitales no puede restringirse solo a la interacción social, pues, hoy en día, está comprobado que facilitan una mejora de la calidad de vida y de la autonomía de la persona. Además, también ha quedado demostrada la necesidad de profundizar en una educación permanente, formación que se debe realizar de manera continua durante toda la vida (Morales, 2013).

Fernández, J.M., Malgor, M., y Rodríguez, M.D. (2021). Nuevas tecnologías, voluntariado y participación activa: tres claves para activar a las personas. RED Visual: Revista Especializada en Discapacidad Visual, 78, 55-68. https://doi.org/10.53094/SNSM7010. 


\section{Fundamentación}

El alfabetismo digital ha sido definido como la capacidad de la persona para localizar información, analizarla, comprenderla y organizarla utilizando las tecnologías digitales.

El vertiginoso avance tecnológico no siempre ha estado unido a la necesaria accesibilidad universal para todos los usuarios. En el caso de las personas afiliadas a la ONCE con ceguera total o déficit visual, fue durante muchos años motivo de lógica preocupación, porque existía el riesgo de que nuestro colectivo, sobre todo usuarios de mayor edad que no estaban familiarizados con las nuevas tecnologías, quedasen fuera de los beneficios y oportunidades que podían conseguirse.

Por ello, durante mucho tiempo, se estuvieron barajando e implementando diferentes estrategias que permitieran el acercamiento y motivación a su utilización por el mayor número de este colectivo, que, por rechazo, desconocimiento o inseguridad corrían el riesgo de no incorporarse a la era digital.

Desde la Delegación Territorial de la Organización Nacional de Ciegos Españoles (ONCE) en Asturias, esta andadura consolidó un grupo de usuarios cada vez más interesados, provocando un aumento de demandas y consultas a los instructores de tiflotecnología y braille (ITB), profesionales especializados en orientar y asesorar sobre aplicaciones que facilitan la accesibilidad a tecnologías y dispositivos técnicos adaptados que mejor se ajusten a la condición visual, intereses y necesidades de las personas afiliadas a la ONCE, así como enseñar el manejo de aquellas en las que deseen o requieran formarse por motivos educativos, laborales, ocio, etc. Estas demandas y consultas estaban basadas, por un lado, en la necesidad de reforzar aprendizajes adquiridos, y, por otro, en necesidades de usuarios más avezados en el uso accesible de aplicaciones o funcionalidades que iban descubriendo.

Esta nueva realidad hizo al equipo plantearse otras estrategias de intervención. El reto era articular un medio que, partiendo de la formación adecuada y básica impartida por los técnicos en tiflotecnología, pudiera tener continuidad con una tutoría práctica de otros usuarios voluntarios más experimentados.

Finalmente, se promocionó la creación de un nuevo espacio colaborativo de apoyo mutuo entre personas afiliadas, dándole forma dentro del marco de actuación del Programa de Voluntariado, diseñando un programa específico de acceso a la información

Fernández, J.M., Malgor, M., y Rodríguez, M.D. (2021). Nuevas tecnologías, voluntariado y participación activa: tres claves para activar a las personas. RED Visual: Revista Especializada en Discapacidad Visual, 78, 55-68. https://doi.org/10.53094/SNSM7010. 
de refuerzo en aprendizaje de tiflotecnología recogido por la normativa interna de la ONCE.

Para su buen funcionamiento, se consideró imprescindible la coordinación con las áreas de Tiflotecnología y de Animación Sociocultural que aportaban las importantes dimensiones formativas y participativas imprescindibles para el buen funcionamiento y desarrollo del programa específico.

Solo quedaba ponerlo en marcha, organizando desde el área de tiflotecnología el ordenado acceso de los primeros participantes, y publicitarlo desde las actividades de animación.

En abril de 2017 se inició el recorrido con 10 usuarios, formando un grupo de WhatsApp que ha ido evolucionando y creciendo hasta llegar al momento actual, en el que un promedio de más de 60 personas con diferentes destrezas en el uso del móvil participan activamente, apoyados por dos voluntarios también afiliados.

\section{Objetivos}

1. Universalizar el uso de las nuevas tecnologías entre afiliados a la ONCE, independientemente de su edad, ubicación geográfica o conocimiento (especialmente del smartphone, por tratarse de la herramienta que está más al alcance de la mayoría de las personas).

2. Brindar espacios informativos sobre aplicaciones útiles facilitadoras para la vida diaria de personas con ceguera total o déficit visual.

3. Detectar nuevas necesidades formativas surgidas en el desarrollo del grupo que permitan mantener actualizadas las demandas de conocimiento y organizar espacios formativos de interés.

4. Crear un espacio cooperativo de apoyo y ayuda mutua en la resolución de dudas y búsqueda de soluciones rápidas.

5. Fomentar el sentimiento de pertenencia a la organización respondiendo a necesidades reales del colectivo. 
6. Empoderar a los afiliados de mayor edad y menos conocimientos tecnológicos, eliminando el rechazo generado por el desconocimiento en el uso de herramientas digitales.

7. Fomentar soluciones interdisciplinares aprovechando sinergias en las diferentes áreas del servicio (animación sociocultural, tiflotecnología, voluntariado), mejorando la comunicación y coordinación entre profesionales.

8. Evitar el aislamiento de afiliados alejados geográficamente, residentes en zonas rurales, conectándolos a los servicios mediante el grupo.

\section{Población destinataria de la experiencia}

Inicialmente, la actividad se planteó para el colectivo de mayores de 55 años que, previamente, hubiese sido dado de alta en formación básica por los instructores tiflotécnicos y que desearan reforzar el aprendizaje de los denominados «gestos básicos», acciones táctiles concretas que permiten llevar a cabo funciones esenciales que posibilitan el uso de WhatsApp, correo electrónico, uso de agenda de contactos y notas.

Sin embargo, durante estos tres años de evolución nos hemos encontrado que la pertenencia a este grupo resulta útil para todo el colectivo usuario de móviles, por tratar temas que, por su aplicabilidad, resultan de interés general. Es por ello que, como decíamos con anterioridad, en la actualidad hemos pasado de los 10 usuarios iniciales a 60 contabilizados a 30 de octubre del 2020, en edades que oscilan entre los 24 años, el más joven, y los 90, el de edad más avanzada, aunque la inmensa mayoría siguen siendo los mayores de 55 años, como se describe a continuación:

- Menores de 55 años: 22 afiliados (36,7\%).

- Edad comprendida entre 56 y 65: 23 afiliados (38,3\%).

- Edad comprendida entre 66 y 75: 12 afiliados (20\%).

- Mayores de 76: 3 afiliados (5\%).

Eso significa que $38(63,3 \%)$ son personas afiliadas mayores de 55 años.

Fernández, J.M., Malgor, M., y Rodríguez, M.D. (2021). Nuevas tecnologías, voluntariado y participación activa: tres claves para activar a las personas. RED Visual: Revista Especializada en Discapacidad Visual, 78, 55-68. https://doi.org/10.53094/SNSM7010. 


\section{Temporalización}

\subsection{7-2018}

El grupo formado por 9 afiliados (5 mujeres y 4 hombres) en edades comprendidas entre 58 y 80 años, inicia su andadura en abril del 2017 con la colaboración de un voluntario y carácter experimental.

En la revisión realizada en noviembre de ese mismo año ya se incorporaron 4 nuevos afiliados (una mujer y tres hombres de edades comprendidas entre los 56 y 73 años), materializándose el ofrecimiento como voluntario de otro afiliado con perfil idóneo para colaborar activamente en la marcha de la iniciativa.

En ese primer año se mantienen las siguientes acciones:

- Reuniones informativas previas con afiliados, detectados por el servicio de tiflotecnología, que han realizado acciones formativas básicas para el manejo de teléfonos móviles con la finalidad de animarlos a incorporarse al grupo.

- Refuerzo de los aprendizajes adquiridos por usuarios de nivel básico: hacer y recibir llamadas, uso de servicios de mensajería, notas de voz, modificación de ajustes.

- Intercambio de información y de estrategias como usuarios entre los miembros del grupo mediante el uso de una carpeta compartida en Google Drive, en la que se almacenan distintos contenidos organizados en categorías, principalmente audioguías, demostraciones y tutoriales, otorgando permisos al ITB, que también genera material.

- Trimestralmente, desde el área de animación se organizó una actividad de dos horas en las que, presencialmente y con la participación de un ITB, se revisaron dudas y se pusieron en común prácticas consolidadas. Ese mismo espacio sirvió para detectar intereses y canalizar nuevas demandas de aprendizaje en aplicaciones o herramientas novedosas para los afiliados.

Podría destacarse una yincana tecnológica en la que los participantes, usando WhatsApp, localizaban lugares a los que debían llegar y enviaban fotos que acreditaran su descubrimiento (WhatsPaseando).

Fernández, J.M., Malgor, M., y Rodríguez, M.D. (2021). Nuevas tecnologías, voluntariado y participación activa: tres claves para activar a las personas. RED Visual: Revista Especializada en Discapacidad Visual, 78, 55-68. https://doi.org/10.53094/SNSM7010. 


\subsection{8-2019}

Se amplía y extiende el grupo con la incorporación y participación activa de nuevos usuarios, extendiéndose la actividad trimestral presencial también a las agencias, permitiendo la relación personal entre afiliados y dando continuidad a las formaciones recibidas.

Durante este período destacaríamos las siguientes actividades:

- «Aplicaciones paso a paso». Talleres presenciales sobre Google Maps, Be My Eyes, Apolo y GOLD.

- Scape room accesible organizado en mayo del 2019, en el que los participantes usaron códigos QR, aplicaciones OCR y redes sociales.

- Asistentes virtuales y domótica, con la información y demostración de las utilidades que, para el uso en el hogar, podían ofrecer Google Home y Amazon Alexa.

- Pódcast, como nueva herramienta de comunicación, con información sobre plataformas accesibles, programas con mayor audiencia en español y herramientas para la creación propia.

Paralelamente, se incorpora como sistema de evaluación y seguimiento la cumplimentación, por parte de los participantes, en Formularios de Google de cuestionarios de satisfacción.

\subsection{9-2020}

Este fue el período de madurez y consolidación del grupo. Se mantiene la misma dinámica de reuniones mensuales hasta la llegada del estado de alarma, en el que el grupo liderado por los voluntarios toma la iniciativa para iniciarse en los contactos por plataformas digitales accesibles valoradas previamente por el ITB, como Zoom o Jitsi Meet, obligando a los participantes a iniciarse en el conocimiento de estos sistemas de comunicación.

Ejemplo de ello es la actividad «Vamos de safari», organizada los días 26 de marzo y 3 de abril, que consistió en una descripción guiada por pantallas del navegador del

Fernández, J.M., Malgor, M., y Rodríguez, M.D. (2021). Nuevas tecnologías, voluntariado y participación activa: tres claves para activar a las personas. RED Visual: Revista Especializada en Discapacidad Visual, 78, 55-68. https://doi.org/10.53094/SNSM7010. 
iPhone para que usuarios sin conocimientos previos pudiesen aprender a utilizarlo, o la celebración del tercer aniversario del grupo el sábado 26 y domingo 27 del mismo mes, días en los que los usuarios del grupo podían incorporarse a las actividades programadas en horarios de encuentro, en las que se compartieron charlas y audios de teatro y de coros vinculados con la ONCE.

La actividad formativa y de refuerzo con voluntarios se retomó a principios de octubre de este año, publicitando, en la primera semana de ese mes, sesiones de aprendizaje sobre el acceso a la plataforma Zoom en grupos de cuatro o cinco personas, con la finalidad de que llegaran a familiarizarse en su uso y así acceder a las actividades de animación online programadas para el trimestre.

\section{Metodología}

La metodología es totalmente participativa colaborativa, desarrollándose de manera sistemática con la clara intención de fomentar en los afiliados con diferentes niveles de aprendizaje y experiencia la adquisición de conocimientos básicos con los que poder utilizar de manera autónoma el smartphone.

La finalidad es que todos los miembros del grupo acaben gestionando las aplicaciones que necesiten en función de sus necesidades y grado o nivel de aprendizaje y uso, proporcionando conocimientos necesarios para nuevas posibilidades (hogar inteligente, orientación, transporte, banca, compras, combinación de dispositivos...).

El hecho de que puedan interactuar usuarios con diferentes niveles de conocimiento, desde los más expertos hasta los recién iniciados -ofreciendo los primeros el apoyo mutuo necesario para el refuerzo en el aprendizaje de gestos básicos y la solución de dificultades que, como consecuencia de la deficiencia visual, requieren el manejo de herramientas de accesibilidad específicas-, funciona, en la mayoría de casos, como un mecanismo motivante en el que poder reflejarse e identificarse.

Los participantes han llegado a plantear necesidades de formación, canalizándolas adecuadamente hacia los técnicos responsables del programa, permitiendo avanzar en conocimiento y en estrategias de resolución de necesidades.

Fernández, J.M., Malgor, M., y Rodríguez, M.D. (2021). Nuevas tecnologías, voluntariado y participación activa: tres claves para activar a las personas. RED Visual: Revista Especializada en Discapacidad Visual, 78, 55-68. https://doi.org/10.53094/SNSM7010. 
Es importante destacar el alto nivel de compromiso personal en la pertenencia al grupo, ya que las personas son libres para acceder o salir comprometiéndose en el uso responsable del mismo, permitiendo avanzar en la consolidación de este, incorporando nuevos miembros y garantizando la continuidad del proyecto.

Los diferentes técnicos que participan han podido aprovechar las sinergias de cada área y responder de manera dinámica e interdisciplinar a las necesidades planteadas por los afiliados. Esto ha sido posible gracias al método colaborativo desarrollado, cuyos principales puntos exponemos a continuación:

- Establecimiento de un proceso de información y coordinación entre las diferentes áreas, detectando posibles candidatos con los que iniciar la actividad, fijando un proceso riguroso de incorporación al grupo con un mínimo de conocimiento en gestos básicos de uso avalado por los ITB.

- Liderazgo en la publicitación, organización y programación de las reuniones presenciales para el grupo mediante la divulgación de actividades de animación.

- Información y aceptación del voluntario seleccionado y de los afiliados susceptibles de incorporarse: listado inicial de participantes.

- Elaboración del programa específico de voluntariado como acompañamiento en el refuerzo de aprendizaje en el manejo de móviles siguiendo los criterios de la normativa interna de la ONCE.

- Incorporación en el grupo de WhatsApp de un ITB y de la coordinadora de animación como afiliados oyentes, como facilitadores y detectores de necesidades o demandas.

- Preparación del calendario y los contenidos de las reuniones presenciales, teniendo en cuenta los intereses de los participantes.

- Seguimiento de la actividad desde las tres áreas de actuación: animación sociocultural, tiflotecnología y voluntariado.

- Generación de material, suministrado principalmente en audio, mediante la carpeta compartida creada a tal efecto. 
- Coordinación interdisciplinar y reuniones semestrales de evaluación del proyecto.

- Evaluación semestral cuantitativa y cualitativa de satisfacción y resultados.

\section{Resultados}

A 30 de octubre del 2020, el grupo está formado por 60 personas en edades comprendidas entre 24 y 90 años.

De los participantes, el $50 \%$ son mujeres y el $50 \%$ hombres, son ciegos totales 31 personas $(51,7 \%)$ y deficientes visuales $29(48,3 \%)$, repartidos por todo el ámbito geográfico de la Delegación Territorial. Un total de 45 (75\%) en zonas urbanas donde hay una sede de la ONCE, 14 en entornos rurales (23,33\%) y un afiliado residente en el extranjero.

La mayoría de participantes están jubilados: 49 personas ( $81,66 \%) ; 2$ son estudiantes $(3,33 \%)$ y en activo laboralmente se encuentran $9(15 \%)$.

Los niveles de satisfacción manifestado en los cuestionarios de participación cumplimentados nos dicen que el nivel de complacencia es muy alto, corroborado por la permanencia de los miembros del grupo.

\section{Conclusiones}

A través del trabajo multidisciplinar que implica la coordinación de voluntariado, la organización de actividades de ocio y promoción deportiva y la utilización de las nuevas tecnologías, hemos conseguido promover el trabajo en equipo, aumentar la implicación y participación de las personas en actividades socioculturales, promocionar acciones deportivas y facilitar la formación e intercambio de impresiones sobre nuevas tecnologías que mejoren su autonomía personal, con todo lo positivo que ello implica para el ajuste a la discapacidad visual.

La realidad surgida tras la aparición de la covid-19 refuerza nuestra intención de seguir trabajando en esta línea, analizando qué herramientas tecnológicas pueden servir para poner en contacto a las personas de acuerdo a sus intereses y necesidades, mejorando su calidad de vida, promocionando una vida activa y saludable y mejorando el acceso

Fernández, J.M., Malgor, M., y Rodríguez, M.D. (2021). Nuevas tecnologías, voluntariado y participación activa: tres claves para activar a las personas. RED Visual: Revista Especializada en Discapacidad Visual, 78, 55-68. https://doi.org/10.53094/SNSM7010. 
a la información. Por ello, está programada la sesión de «Deportes y tecnología» y previstas otras tres acciones formativas sobre el acceso a las aplicaciones de compras por internet, gestión de trámites administrativos y dispositivos inteligentes en el hogar.

La instrucción no puede ni debe quedarse únicamente en el manejo de las principales herramientas de adaptación, sino que pretendemos acercar servicios accesibles del día a día que muchas personas desconocen, permitiéndoles tener una vida más integrada y comunicada, continuando con la satisfactoria experiencia de unir las nuevas tecnologías, el servicio de voluntariado y el aprovechamiento del ocio y el tiempo libre.

\section{Agradecimiento}

No podemos finalizar esta exposición, sin manifestar nuestro reconocimiento a todos los profesionales que a lo largo de estos años se han implicado en la implementación y desarrollo de esta actividad, así como a la labor desinteresada y altruista de los voluntarios sin cuyo esfuerzo y dedicación no se habrían podido alcanzar los resultados actuales.

\section{Referencias bibliográficas}

Fernández, M., Chipre, L., y Vidal P. (2016). Las TIC: una oportunidad para la inclusión [PDF]. Observatorio de la Discapacidad Física.

García-Valcárcel, A., Hernández, A., y Recamán, A. (2012). La metodología del aprendizaje colaborativo a través de las TIC: una aproximación a las opiniones de profesores y alumnos [PDF]. Revista Complutense de Educación, 23(1), 161-188. https://doi. org/10.5209/rev_RCED.2012.v23.n1.39108.

Llorente-Barroso, C., Viñarás-Abad, M., y Sánchez-Valle, M. (2015). Mayores e Internet: la Red como fuente de oportunidades para un envejecimiento activo [PDF]. Comunicar: Revista Científica de Comunicación y Educación, 23(45), 29-36. http://dx.doi. org/10.3916/C45-2015-03.

Morales, P. (2013). El uso de las TIC y la formación permanente del adulto: una mejora de la calidad de vida [PDF]. Ariadna; cultura, educación y tecnología, 1(1), 58-62. http:// dx.doi.org/10.6035/Ariadna.2013.1.9.

Fernández, J.M., Malgor, M., y Rodríguez, M.D. (2021). Nuevas tecnologías, voluntariado y participación activa: tres claves para activar a las personas. RED Visual: Revista Especializada en Discapacidad Visual, 78, 55-68. https://doi.org/10.53094/SNSM7010. 
Pérez, G., y Sarrate, M. L. (2011). Las TIC promotoras de inclusión social [PDF]. Revista española de pedagogía, 249, 237-253.

Piattini, M. (2012). Papel de las TIC en el envejecimiento [PDF]. Lychnos, 8, 60-64.

José Manuel Fernández Granda. Instructor en tiflotecnología y braille. Delegación Territorial de la ONCE en Asturias. Calle de Campomanes, 9; 33008 Oviedo (España). Correo electrónico: jofegr@once.es.

Marta Malgor. Coordinadora de Animación Sociocultural y Deporte. Delegación Territorial de la ONCE en Asturias. Calle de Campomanes, 9; 33008 Oviedo (España). Correo electrónico: mamva@once.es.

María Dolores Rodríguez Miranda. Trabajadora social. Delegación Territorial de la ONCE en Asturias. Calle de Campomanes, 9; 33008 Oviedo (España). Correo electrónico: drm@once.es. 